\title{
THE NUMERICAL SOLUTION OF STOCHASTIC DIFFERENTIAL EQUATIONS
}

\author{
P. E. KLOEDEN and R. A. PEARSON
}

(Received 14 September 1976)

\begin{abstract}
A method is proposed for the numerical solution of Itô stochastic differential equations by means of a second-order Runge-Kutta iterative scheme rather than the less efficient Euler iterative scheme. It requires the Runge-Kutta iterative scheme to be applied to a different stochastic differential equation obtained by subtraction of a correction term from the given one.
\end{abstract}

It was observed by Wright [8] that different iterative schemes for the numerical solution of stochastic differential equations

$$
d x_{l}=a\left(t, x_{i}\right) d t+b\left(t, x_{t}\right) d \xi_{t},
$$

where $\xi_{t}$ is a Wiener process, converge to different solutions for the same noise sample and initial condition. This is in contrast to their deterministic counterparts for ordinary differential equations, which converge to the same solution.

Strictly speaking stochastic differential equations (1) are really integral equations

$$
x_{i}=x_{0}+\int_{l_{0}}^{t} a\left(s, x_{s}\right) d s+\int_{l_{0}}^{t} b\left(s, x_{s}\right) d \xi_{s},
$$

where the second integral is either an Ito or Stratonovich stochastic integral, in the definitions of which the functions are evaluated, respectively, at the left-hand endpoint and midpoint of each partition subinterval (for example, [4], [6], [7, chapter 3]). Consequently the Euler iterative scheme

$$
x_{n+1}=x_{n}+a\left(t_{n}, x_{n}\right) h+b\left(t_{n}, x_{n}\right) \xi_{n}
$$

converges to a sample path of the Ito solution of (2) whereas the second-order Runge-Kutta iterative scheme

$$
\begin{aligned}
x_{n+1}=x_{n} & +\frac{1}{2}\left\{a\left(t_{n}, x_{n}\right)+a\left(t_{n+1}, x_{n}+a\left(t_{n}, x_{n}\right) h+b\left(t_{n}, x_{n}\right) \xi_{n}\right)\right\} h \\
& +\frac{1}{2}\left\{b\left(t_{n}, x_{n}\right)+b\left(t_{n+1}, x_{n}+a\left(t_{n}, x_{n}\right) h+b\left(t_{n}, x_{n}\right) \xi_{n}\right)\right\} \xi_{n}
\end{aligned}
$$

converges to a sample path of the Stratonovich solution of (2). In these iterative schemes a partition $t_{0}<t_{1}<\ldots<t_{N}$ is used with uniform step length $h=t_{i+1}-t_{i}$ and an $N\left(0, h^{\frac{1}{2}}\right)$ distributed noise sample $\xi_{0}, \xi_{1}, \ldots, \xi_{N-1}$, where $\xi_{i}$ represents the 
change in noise between points $t_{i}$ and $t_{i+1}$. The above convergences can be proved by similar arguments to those in Theorems 8.1 and 11.1 of [4]. Moreover, it can be shown by straightforward but tedious error analysis, as in [5], that the Euler iterative scheme (3) is of order $O_{p}\left(h^{\mathbf{l}}\right)$ and the second-order Runge-Kutta iterative scheme (4) is of order $O_{p}\left(h^{\natural}\right)$, where $O_{p}\left(h^{\nu}\right)$ for some $\nu>0$ means

$$
\lim _{\mathrm{h} \rightarrow 0} h^{-\nu} \operatorname{Prob}\{\mid \text { error } \mid \geqslant \varepsilon\}=0
$$

for all $\varepsilon>0$. The fundamental feature of such error analysis is that for such noise changes $\xi_{i}$ as above

$$
E\left|\xi_{i}\right|^{m} \leqslant \text { const } h^{\frac{1}{\mathbf{b} m}}
$$

for $m=1,2,3, \ldots$

Most applications of stochastic differential equations use the Itô solution as it is a Markov process. Consequently, the Euler iterative scheme is used to obtain numerical solutions (for example, [1]). The second-order Runge-Kutta iterative scheme (4) would be more efficient, but leads to the Stratonovich solution rather than the required Itô solution. However, the Itô solution of (2) coincides with the Stratonovich solution of a different stochastic integral equation

$$
x_{t}=x_{0}+\int_{t_{0}}^{t}\left\{a\left(s, x_{s}\right)-\frac{1}{2} b\left(s, x_{s}\right) \frac{\partial b}{\partial x}\left(s, x_{s}\right)\right\} d s+\int_{t_{0}}^{t} b\left(s, x_{s}\right) d \xi_{s} .
$$

This follows from the relationship

$$
\text { (S) } \int_{l_{0}}^{t} b\left(s, x_{s}\right) d \xi_{s}=(I) \int_{t_{0}}^{t} b\left(s, x_{s}\right) d \xi_{s}+\frac{1}{2} \int_{l_{0}}^{t} b\left(s, x_{8}\right) \frac{\partial b}{\partial x}\left(s, x_{s}\right) d s
$$

between the Stratonovich and Itô stochastic integrals [6, equation $\left.7^{\prime}\right]$. The secondorder Runge-Kutta iterative scheme applied to (5) will converge to a sample path of the Stratonovich solution of (5) or equivalently to a sample path of the Itô solution of (2). Hence it is proposed that in order to solve (2) numerically for its It $\hat{o}$ solution, the second-order Runge-Kutta iterative scheme for (5) should be used.

To illustrate the improved efficiency offered by this proposal, a sample path with initial condition $x_{0}=1$ of the Itô solution of the stochastic differential equation

$$
d x_{t}=x_{t} d \xi_{t}
$$

was determined numerically by both the Euler iterative scheme for (6) and the second-order Runge-Kutta iterative scheme for

$$
d x_{i}=-\frac{1}{2} d t+x_{i} d \xi_{i}
$$

over the time interval $0 \leqslant t \leqslant 1$. Equation (6) was chosen because its Itô solution is known analytically, namely

$$
x_{i}=\exp \left(\xi_{i}-\frac{1}{2} t\right)
$$


In order to compare convergence rates the calculations were repeated for successively finer approximations to the same noise sample by successively doubling the number of partition subintervals in such a way that the value of an approximation to the Wiener process $\xi(t)$ at any partition point $t_{i}$ equalled the values of finer approximations at $t_{i}$. The computed values of the sample path at time $t=1$ from both schemes are plotted against the number of partition subintervals in Fig. 1 and are compared with the analytic solution (8) calculated with the approximation to the Wiener process at time $t=1$. In Fig. 2 the logarithm of the error for each

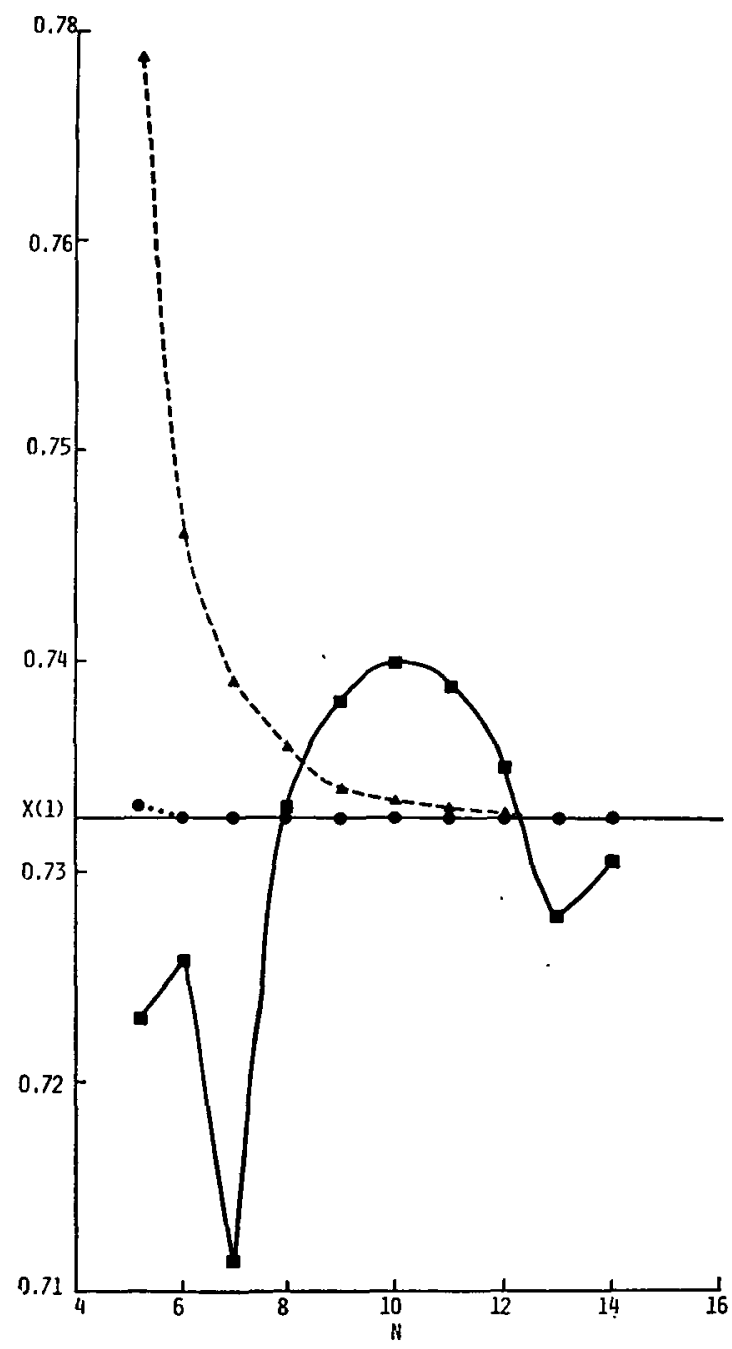

Fig. 1. The solution at $t=1, h=\frac{1}{2}^{n}$. ——_, Euler method; $\Delta-\ldots-$, Runge-Kutta second order; . . . Runge-Kutta fourth order with Gill's coefficients. 


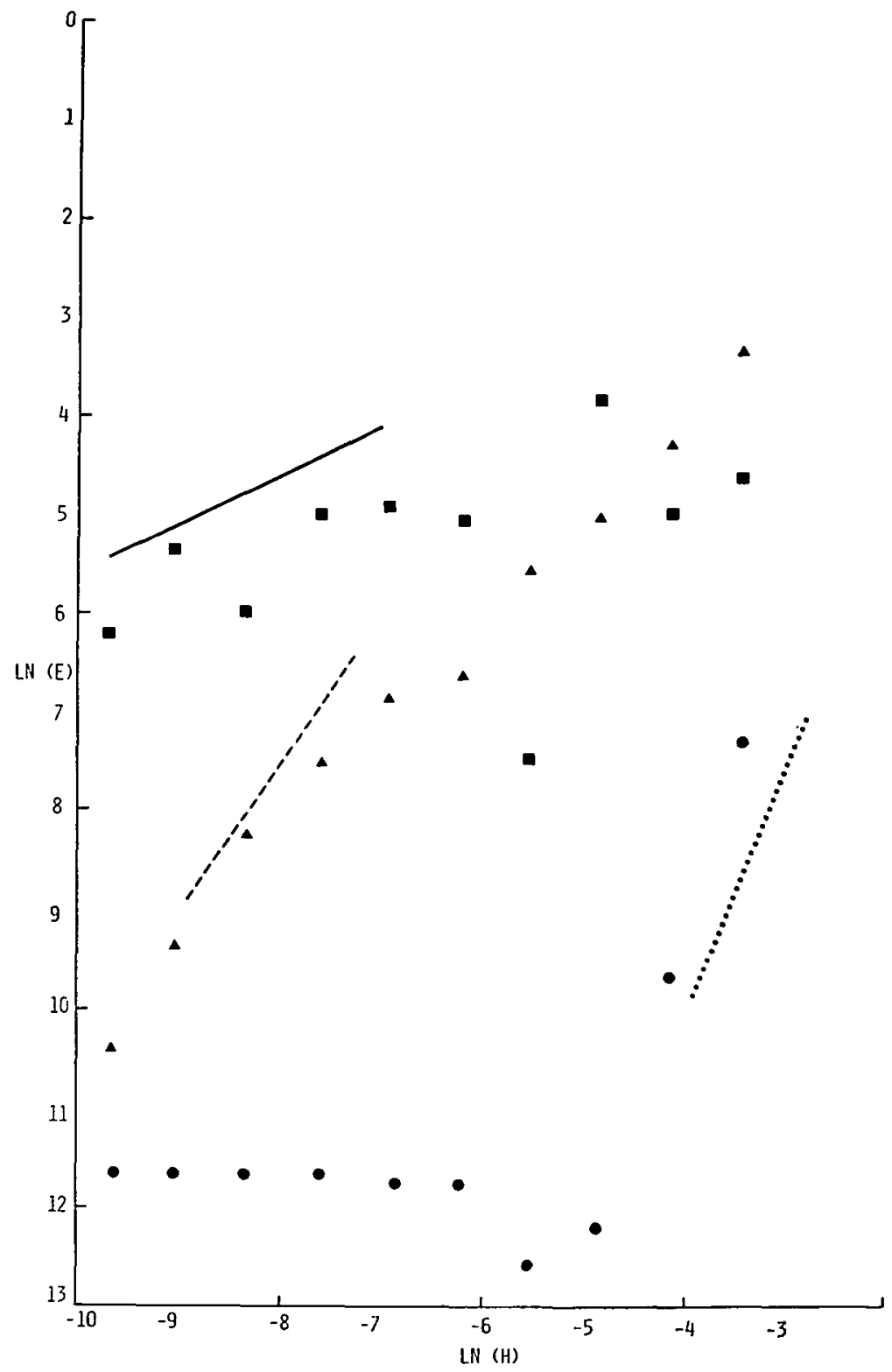

Fig. 2. The error in the solution. $\square$, Euler method; $\boldsymbol{A}$, Runge-Kutta second order; $\bullet$, RungeKutta fourth order with Gill's coefficients; corresponds to an error $O_{h}\left(h^{t}\right)$; --- , corresponds to an error $O_{p}\left(h^{i}\right)$; $\cdots$, corresponds to an error $O_{p}\left(h^{l}\right)$. 
scheme is plotted against the logarithm of the step length $h$. The graphs of the Euler and second-order Runge-Kutta iterative schemes have approximate slopes $\frac{1}{2}$ and $\frac{3}{2}$, respectively, which is in accord with their respective error bounds of $O_{p}\left(h^{\mathbf{t}}\right)$ and $O_{p}\left(h^{\frac{3}{2}}\right)$.

The two figures also include analogous results for the fourth-order Runge-Kutta iterative scheme with Gill coefficients (for example, [2]) applied to equation (8). This seems to converge to the Stratonovich solution of (8) with error bound $O_{\hat{p}}\left(h^{t}\right)$ (the flattening out of its graph in Fig. 2 is due to rounding off the irrational Gill coefficients). This and other fourth-order Runge-Kutta iterative schemes (for example, [2], [3], [8]) converge much more rapidly than the second-order RungeKutta iterative scheme, but it has not yet been theoretically established to which solution of a stochastic differential equation they converge. For instance, it was found that the fourth-order Runge-Kutta iterative schemes with Runge [2] or Kutta [3] coefficients applied to equation (8) did not converge to the Stratonovich solution of equation (8).

\section{References}

[1] D. J. Clements and B. D. O. Anderson, "Well-behaved Itô equations with simulations always misbehaved", IEEE Trans. Automatic Control AC-18 (1973), 676-677.

[2] P. Henrici, Discrete Variable Methods in Ordinary Differential Equations, John Wiley and Sons, New York (1965).

[3] S. S. Kuo, Computer Applications of Numerical Methods, Addison-Wesley, Reading, Mass. (1972).

[4] E. J. McShane, "Stochastic differential equations and inodels of random processes", in Proc. Sixth Berkeley Symp. Probability and Mathematical Statistics (1971) 263-294.

[5] N. J. Rao, J. D. Borwankar and D. Ramkrishna, "Numerical solution of Itô integral equations", SIAM J. Control 12 (1974), 124-139.

[6] R. L. Stratonovich, "A new representation for stochastic integrals and equations", SIAM J. Control 4 (1966), 362-371.

[7] E. Wong, Stochastic Processes in Information and Dynamical Systems, McGraw-Hill, New York (1971).

[8] D. J. Wright, "The digital simulation of stochastic differential equations," IEEE Trans. Automatic Control AC-19 (1974), 75-76.

Department of Mathematics

Monash University

Clayton, Vic. 3168

Australia 